\title{
NOVA ABORDAGEM SOBRE O MODELO BRASILEIRO DE SERVIÇOS AMBIENTAIS ${ }^{(1)}$
}

\author{
Dulce Buchala Bicca Rodrigues ${ }^{(2)}$, Teodorico Alves Sobrinho ${ }^{(3)}$, \\ Paulo Tarso Sanches de Oliveira ${ }^{(4)}$ \& Elói Panachuki ${ }^{(5)}$
}

\begin{abstract}
RESUMO
O programa brasileiro de serviços ambientais, denominado "Produtor de Água", apoia-se em incentivos financeiros proporcionais à redução da erosão advinda da implantação voluntária de projetos de conservação do solo por parte de produtores rurais. No entanto, esse programa não constitui restrições preestabelecidas de elegibilidade de áreas de aplicação ou de propostas de conservação. Assim, no sentido de contribuir para a evolução metodológica desse programa, propõe-se uma nova abordagem deste, analisando-se, essencialmente, duas adaptações: introdução de critério de elegibilidade; e utilização da aptidão agrícola para indicação de propostas de uso intensivo e conservação do solo. Para isso, tomou-se por base uma bacia hidrográfica rural próxima ao Pantanal brasileiro, em Terenos, MS. O novo critério de elegibilidade foi baseado no Risco Natural de Erosão (razão entre o potencial natural de erosão e a tolerância à perda de solo). As propostas de ocupação do solo, por sua vez, foram definidas a partir de mapa de aptidão agrícola predefinido. $O$ estudo revela que o novo critério de elegibilidade foi capaz de contemplar somente cerca de 40 \% da área de estudo. A aplicação da classificação de aptidão agrícola nessa parcela reduziu drasticamente as alternativas de uso intensivo do solo, direcionando-as, em grande parte, para a atividade pecuária. A análise das duas propostas de inovação do programa "Produtor de Água" permite inferir quanto à potencialidade delas na formação de estratégias de aplicação do programa, além de promover uso mais eficiente dos recursos financeiros destinados ao pagamento por serviços ambientais no contexto brasileiro.
\end{abstract}

Termos de indexação: uso sustentável do solo, conservação do solo, políticas agroambientais.

\footnotetext{
(1) Parte da Dissertação de Mestrado do primeiro autor. Recebido para publicação em setembro de 2010 e aprovado em março de 2011.

(2) Doutoranda em Engenharia Hidráulica e Saneamento, Universidade de São Paulo - USP. Caixa Postal 359 , CEP $13560-970$ São Carlos (SP). E-mail: dulce@sc.usp.br

(3) Professor Associado III, Universidade Federal de Mato Grosso do Sul - CCET/UFMS. Caixa Postal 549, CEP 79070-900 Campo Grande (MS). E-mail: talves@ufms.br

(4) Doutorando em Engenharia Hidráulica e Saneamento, USP E-mail: paulotarsoms@gmail.com

(5) Professor da Universidade Estadual de Mato Grosso do Sul - UEMS. Caixa Postal 25, CEP 79200-000 Aquidauana (MS). Email: eloip@uems.br
} 


\title{
SUMMARY: NEW APPROACH TO THE BRAZILIAN MODEL OF ENVIRONMENTAL SERVICES
}

\begin{abstract}
The Brazilian program of environmental services, called "Water Supplier", is based on financial incentives relative to the erosion reduction by the implementation of voluntary soil conservation projects by farmers. However, this program has no pre-established restrictions of eligibility of application areas or conservation proposals. With a view to contribute to the methodological development of this program, a new approach was proposed, by analysis of two main changes: introduction of eligibility criteria, and the use of land suitability for recommendation of proposals for intensive soil use and conservation. For this purpose, a rural catchment was studied near the Brazilian Pantanal, in Terenos, MS. The new eligibility criteria were based on the natural erosion risk (ratio of natural erosion potential and soil loss tolerance). The land use proposals, in turn, were based on a pre-defined land suitability map. This study reveals that the new eligibility criteria covered only about $40 \%$ of the study area. The classification of land suitability in this area reduced the intensive land use alternatives dramatically, indicating it largely for cattle ranching. The analysis of the two innovation proposals of the program "Water Supplier" allowed inferences on their potential to generate strategies for implementing the program, and to promote a more efficient use of financial resources for payment for environmental services in the Brazilian context.
\end{abstract}

Index terms: sustainable land use, soil conservation, agro-environmental policies.

\section{INTRODUÇÃO}

A mitigação de impactos negativos das atividades agrícolas em bacias hidrográficas pode ser incentivada pelo Pagamento por Serviços Ambientais (PSA), realizado por usuários de água a jusante, ou por instituições governamentais que contribuem voluntariamente para o custeio de práticas de conservação promovidas a montante (Engel et al., 2008). Os serviços ambientais representam os benefícios que a população desfruta, a partir de funções do ecossistema (Costanza et al., 1997, 1998; Chen et al., 2009). O PSA apoia-se no princípio do provedorrecebedor, em substituição ao tradicional poluidorpagador (Kosoy et al., 2007; Engel et al., 2008).

Os Estados Unidos implementam, desde 1985, um programa de PSA, denominado Conservation Reserve Program (CRP), que promove incentivo econômico para práticas de conservação do solo em propriedades rurais (FSA, 1985). Foram diagnosticadas reduções significativas em despesas no processo de tratamento da água proveniente dos mananciais inseridos em parte das áreas contempladas pelo programa CRP, devido ao controle da poluição difusa (Postel \& Thompson, 2005).

O programa CRP utiliza a Universal Soil Loss Equation (USLE) (Wischmeier \& Smith, 1978) para estimativa da perda de solo e prioriza sua aplicação nas áreas classificadas como Altamente Erodíveis (AE), definidas por meio da razão entre o potencial natural de erosão e os valores de tolerância à perda de solo (FSA, 1985; Renschler \& Harbor, 2002; Lant et al., 2005; USDA-NRCS, 2006). Essa relação também pode ser denominada de Risco Natural de Erosão - RNE
(Basic et al., 2004; Boellstorff \& Benito, 2005; Schiettecatte et al., 2008; Oliveira et al., 2011). Assim, o índice RNE é utilizado como critério de elegibilidade para aplicação do programa de serviços ambientais (CRP). Vale ressaltar que a distribuição das áreas classificadas como AE integra parte dos critérios para implementação do programa CRP, prevendo-se outros requisitos a serem atendidos pelos usuários dessas áreas para serem contemplados com os incentivos econômicos do programa (USDA-NRSC, 2006).

O modelo USLE, apesar de algumas limitações quantitativas, ainda é o modelo de erosão mais utilizado mundialmente, fornecendo informações úteis no planejamento conservacionista do solo e água. Caracteriza-se por estabelecer estimativas de perda média anual de solo por erosão laminar e em sulcos (Kinnell, 2010; Oliveira et al., 2011). Os dados de entrada do modelo USLE são compostos por fatores inerentes (erosividade - R; erodibilidade - K; comprimento de rampa - L; e declividade - S) e antrópicos (uso e manejo do solo - C; e práticas conservacionistas - P). A tolerância à perda de solo, por sua vez, pode ser definida como a máxima taxa de erosão do solo que permite a manutenção de elevada capacidade produtiva, viável economicamente e por longo período de tempo (Wishmeier \& Smith, 1978). A tolerância T, se ultrapassada, é considerada inaceitável e deve ser reduzida por meio de medidas de conservação viáveis (Sparovek \& De Maria, 2003; Bühlmann et al., 2010).

No Brasil, o programa de serviços ambientais "Produtor de Água", desenvolvido pela Agência Nacional de Águas, também utiliza o modelo USLE de modo simplificado, apoiando-se apenas na variação 
dos fatores antrópicos $(\mathrm{C}$ e $\mathrm{P})$ para determinação dos valores de PSA. Assim, estima-se o Percentual de Abatimento da Erosão (PAE) a partir dos cenários de redução dos fatores $\mathrm{C} \mathrm{e} \mathrm{P}$; então, adequa-se o valor de PSA por unidade de área, conforme o enquadramento dos valores de PAE. O programa brasileiro apoia-se, essencialmente, em incentivos financeiros proporcionais aos benefícios ambientais relacionados à redução da erosão advinda da implantação de projetos de conservação. O programa objetiva primordialmente a melhoria da qualidade da água e o aumento das vazões médias dos rios em bacias hidrográficas de importância estratégica para o País. É um programa de adesão voluntária de produtores rurais que se proponham a adotar práticas e manejos conservacionistas (Chaves et al., 2004; ANA, 2008; ANA, 2011). Vale ressaltar que a Política Nacional dos Serviços Ambientais está em formulação por meio do Projeto de Lei 5.487/09 (Brasil, 2009).

Apesar de os incentivos econômicos serem uma ferramenta poderosa na conservação ambiental, a determinação do potencial agrícola do solo para diferentes tipos de utilização é fundamental para o desenvolvimento de atividades agrícolas em bases sustentáveis (Costa et al., 2008). Nesse contexto, a classificação da aptidão agrícola fornece opções de uso cuja escolha deve considerar a viabilidade socioeconômica, a legislação ambiental e o interesse do produtor (Menezes et al., 2009). O cruzamento de mapas de aptidão agrícola do solo com mapas de uso do solo atual permite a verificação da adequabilidade do uso preponderante (Pedron et al., 2006), bem como do nível de utilização do solo (Gomes et al., 2007). O sistema de avaliação da aptidão agrícola proposto por Ramalho Filho \& Beek (1995) tem sido o método mais empregado, por constituir um importante instrumento para conhecer o potencial e a disponibilidade de área de acordo com diferentes níveis de tecnologia ou de manejo (Chagas et al., 2006).

Assim, de forma a contribuir para a evolução metodológica do programa brasileiro de serviços ambientais, propõe-se uma nova abordagem dele, analisando, essencialmente, duas adaptações: introdução de critério de elegibilidade; e utilização da aptidão agrícola para indicação de propostas de uso intensivo e conservação do solo.

\section{MATERIAL E MÉTODOS}

\section{Área de estudo}

O estudo foi realizado tomando-se por base a bacia hidrográfica do ribeirão Salobra (Terenos, MS), com aproximadamente $540 \mathrm{~km}^{2}$, situada entre as coordenadas de $20^{\circ} 12^{\prime} \mathrm{S}$ a $20^{\circ} 28^{\prime} \mathrm{S}$ e de $54^{\circ} 55^{\prime} \mathrm{W}$ a $55^{\circ} 16^{\prime} \mathrm{W}$, próxima aos limites dos biomas Cerrado e Pantanal (Figura 1). O ribeirão Salobra é tributário do rio Aquidauana - importante curso d'água para suprimento das atividades pesqueira e agrícola na região.

A área de estudo está localizada na região de planalto da bacia do Alto Paraguai (BAP), sendo de considerável relevância ecológica, pois os problemas ambientais da região do Pantanal, em parte, dependem dos processos ocorridos no planalto (Wantzen et al., 2008). O Pantanal é reconhecido como a maior planície alagável do mundo e designada como patrimônio nacional pela Constituição Brasileira (Seidl et al., 2001).

O clima da área de estudo é tropical, transição entre Cfa e Aw, de acordo com a classificação de Köppen, com precipitação pluvial média anual de $1.500 \mathrm{~mm}$ e temperatura média anual de $23^{\circ} \mathrm{C}$. A altitude do local varia de 200 a $400 \mathrm{~m}$.

A ocupação da bacia do ribeirão Salobra é rural, predominando a atividade pecuária extensiva e, em menor percentual, culturas lenhosas, como eucalipto e citricultura. A classificação do uso e cobertura do solo foi realizada por meio de imagens Landsat Thematic Mapper (órbita/ponto 225/74) (INPE, 2008).

\section{Adaptações do programa brasileiro de serviços ambientais}

A presente proposta de inovação do programa "Produtor de Água" avaliou duas adaptações principais: introdução de critério de elegibilidade; e utilização da aptidão agrícola para indicação de propostas de uso intensivo e conservação do solo. O contexto de inserção dessas modificações está esquematizado na figura 2.

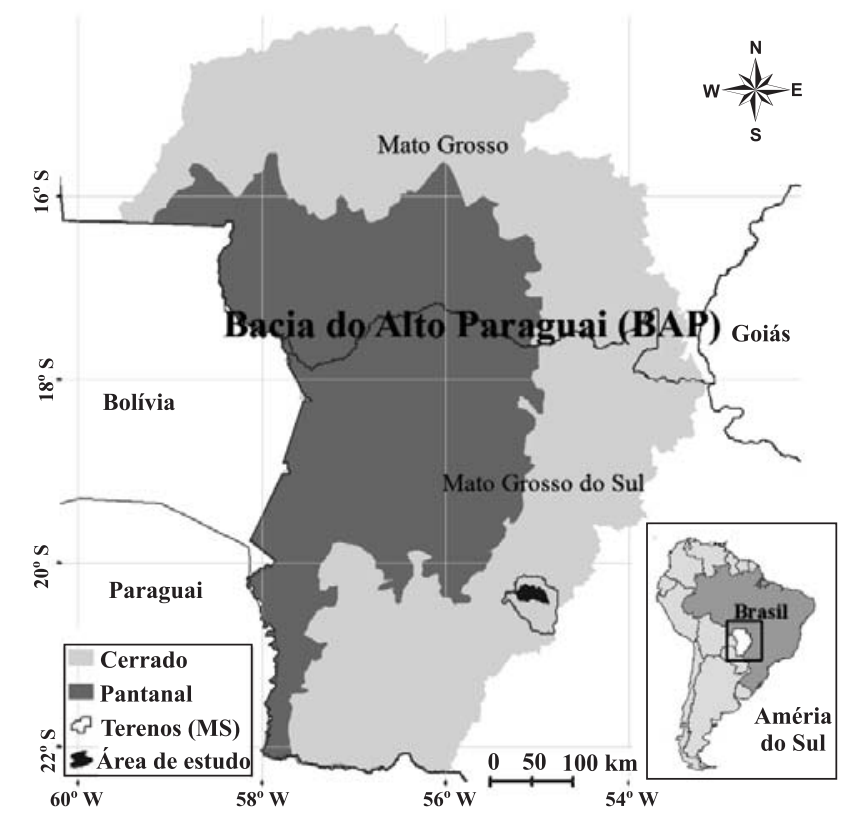

Figura 1. Localização da área de estudo. 


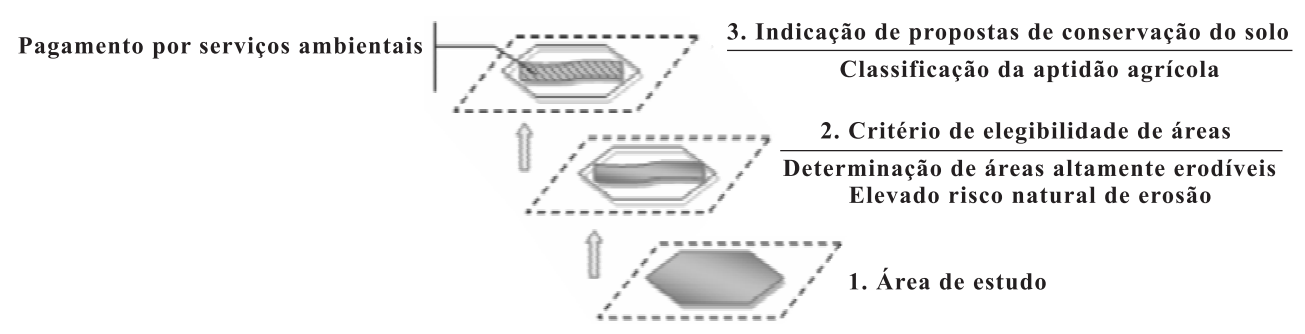

Figura 2. Delineamento do estudo da nova abordagem do programa brasileiro de serviços ambientais.

\section{Critério de elegibilidade}

O novo critério de elegibilidade considerou o índice de Risco Natural de Erosão (RNE), representado pela relação entre o Potencial Natural de Erosão (PNE) e a tolerância à perda de solo (T). O PNE estima a perda média anual de solo a partir dos fatores inerentes do modelo USLE (equação 1). O modelo USLE, proposto por Wischmeier \& Smith (1978), foi aplicado por meio de estimativas in situ e baseadas em literatura dos fatores que integram o modelo.

$$
\mathrm{RNE}=\mathrm{PNE} / \mathrm{T}=\mathrm{RKLS} / \mathrm{T}
$$

em que RNE = Risco Natural de Erosão (adimensional); $\mathrm{PNE}=$ Potencial Natural de Erosão $\left(\mathrm{t} \mathrm{ha}^{-1} \mathrm{ano}^{-1}\right) ; \mathrm{R}=$ erosividade da chuva ( $\left.\mathrm{MJ} \mathrm{mm} \mathrm{ha}^{-1} \mathrm{~h}^{-1} \mathrm{ano}^{-1}\right) ; \mathrm{K}=$ erodibilidade do solo $\left(\mathrm{t} \mathrm{h} \mathrm{MJ}^{-1} \mathrm{~mm}^{-1}\right)$; LS = fator topográfico (adimensional); $\mathrm{e} \mathrm{T}=$ tolerância à perda de solo (t ha ${ }^{-1}$ ano $\left.^{-1}\right)$.

O critério de elegibilidade foi estabelecido em áreas com valores de $\mathrm{RNE}$ iguais ou maiores que 8, classificadas como altamente erodíveis (AE). As demais áreas, por outro lado, foram classificadas como Não Altamente Erodíveis (N-AE). Esse sistema restritivo de implementação do PSA foi aplicado na área de estudo com base no programa CRP (USDA-NRSC, 2006).

\section{Estimativa dos fatores do modelo USLE}

\section{Fator Erosividade Média Anual - R}

Os dados de precipitação pluvial utilizados na obtenção do índice de erosividade do Estado de Mato Grosso do Sul foram provenientes de estações pluviográficas particulares e de estações pluviométricas, disponibilizadas pelo Sistema de
Informações Hidrológicas da Agência Nacional de Águas (HidroWeb) (Quadro 1).

Os dados das três estações pluviográficas foram utilizados para obtenção de equações de regressão entre o índice de erosividade $\mathrm{EI}_{30}$ e o coeficiente de chuvas proposto por Fournier (1956) e modificado por Lombardi Neto (1977). As equações obtidas foram aplicadas a 109 estações pluviométricas, obtendo-se assim os valores $\mathrm{EI}_{30}$. O mapa do índice de erosividade foi gerado a partir do ajuste ao semivariograma, do processo de validação cruzada e da aplicação da krigagem ordinária. Assim, o mapa de erosividade resultante foi recortado conforme o limite da área em estudo.

\section{Fator topográfico - LS}

O fator LS foi gerado a partir de um Modelo Digital de Elevação (MDE) advindo da vetorialização da base topográfica e processado no software USLE-2D (Desmet \& Govers, 1996), conforme Oliveira et al. (2010). O fator L foi obtido por meio da equação 2, conforme Desmet \& Govers (1996).

$\mathrm{L}_{\mathrm{i}, \mathrm{j}}=\left[\left(\mathrm{A}_{\mathrm{i}, \mathrm{j}}+\mathrm{D}^{2}\right)^{\mathrm{m}+1}-\left(\mathrm{A}_{\mathrm{i}, \mathrm{j}}\right)^{\mathrm{m}+1}\right] /\left[\mathrm{x}_{\mathrm{i}, \mathrm{j}}^{\mathrm{m}} \cdot \mathrm{D}^{\mathrm{m}+2} \cdot(22,13)^{\mathrm{m}}\right]$

em que $\mathrm{L}_{\mathrm{i}, \mathrm{j}}=$ fator de comprimento de vertente de uma célula com coordenadas $(i, j) ; A_{i, j}=$ área de contribuição de uma célula com coordenadas $(i, j)\left(\mathrm{m}^{2}\right)$; $\mathrm{D}=$ tamanho da grade de células $(\mathrm{m}) ; \mathrm{x}_{\mathrm{i}, \mathrm{j}}=$ valor da direção do fluxo; e $\mathrm{m}=$ coeficiente dependente da declividade.

O expoente (m) da equação 2 foi obtido considerando as variações da declividade, sendo: $\mathrm{S}<1 \%(\mathrm{~m}=0,2)$; $1 \% \leq \mathrm{S} \leq 3 \%(\mathrm{~m}=0,3) ; 3<\mathrm{S} \leq 5(\mathrm{~m}=0,4) ; \mathrm{e} \mathrm{S}>5 \%$ $(\mathrm{m}=0,5)$. O cálculo do fator $\mathrm{S}$ baseou-se no algoritmo proposto por Wischmeier \& Smith (1978) (equação 3).

Quadro 1. Série de dados analisados para obtenção do fator de erosividade no Estado de Mato Grosso do Sul (MS)

\begin{tabular}{clcc}
\hline Estação & Local & Periodo (ano) & Quantidade de estações \\
\hline Pluviográficas & Dourados & 8 & 1 \\
& Coxim & 4 & 1 \\
Pluviométricas & Campo Grande & 3 & 1 \\
& Municípios de MS & Superior a 15 & 109 \\
\hline
\end{tabular}




$$
\mathrm{S}=65,41 \operatorname{sen}^{2} \theta+4,56 \operatorname{sen} \theta+0,065
$$

em que: $\theta=$ ângulo em graus da encosta.

\section{Fator de Erodibilidade - K e Tolerância a perda de solo - $\mathbf{T}$}

Os valores do fator de erodibilidade do solo (K) e de tolerância à perda de solo (T) foram definidos a partir de dados de literatura, conforme as classes de solo e características descritas no mapa de solo da área de estudo, escala 1:1.000.000 (Brasil, 1982). As classes de solo identificadas são: Latossolo Roxo distrófico (LRd) (14,8 \%), Latossolo Vermelho-Escuro álico (Led) (32,8 \%) e Neossolo Quartzarênico álico (NQo) (52,4 \%) (Quadro 2).

\section{Propostas de Conservação}

De modo a favorecer o planejamento agrícola e ambiental, foram consideradas as classes de aptidão agrícola correspondentes à área de estudo. A bacia de estudo apresenta as classes de aptidão agrícola expostas no quadro 3.

O grupo de aptidão agrícola identifica o tipo de utilização mais intensivo das terras, ou seja, sua melhor aptidão. Conforme a classificação proposta por Ramalho Filho \& Beek (1995), os grupos 1 a 3 são aptos para lavouras; o grupo 4, indicado para pastagem plantada; o grupo 5, para silvicultura e pastagem natural; e o grupo 6 deve ser destinado à preservação da natureza. O aumento das limitações da região produz a diminuição das alternativas de uso (Figura 3).

Quadro 2. Fator erodibilidade e tolerância à perda de solo, conforme a classe de solo

\begin{tabular}{|c|c|c|}
\hline Classe de solo & Fator de K & $\mathbf{T}$ \\
\hline & $\mathrm{t} \mathrm{h} \mathrm{MJ}^{-1} \mathrm{~mm}^{-1}$ & tha $^{-1}$ ano $^{-1}$ \\
\hline Latossolo Roxo distrófico (LRd) & $0,0128^{(1)}$ & $13,0^{(1)}$ \\
\hline Latossolo Vermelho-Escuro distrófico (LEa) & $0,0175^{(1)}$ & $15,0^{(1)}$ \\
\hline Neossolo Quatzarênico órtico (RQo) & $0,0351^{(2)}$ & $6,3^{(3)}$ \\
\hline
\end{tabular}

Fonte: ${ }^{(1)}$ Oliveira et al. (2007); ${ }^{(2)}$ Silva \& Álvares (2005); ${ }^{(3)}$ Oliveira et al. (2008).

Quadro 3. Descrição das classes de aptidão agrícola observadas na bacia do ribeirão Salobra

\begin{tabular}{cl}
\hline Classificação da aptidão agrícola & \multicolumn{1}{c}{ Descrição } \\
\hline $2(\mathrm{a}) \mathrm{BC}$ & Aptidão regular nos níveis de manejo B e C e restrita no A \\
$3(\mathrm{a})(\mathrm{b})(\mathrm{c})$ & Aptidão restrita para lavoura nos níveis de manejo A, B e C \\
$4(\mathrm{p}) / 5(\mathrm{~s})$ & Aptidão restrita para pastagem plantada e silvicultura \\
\hline
\end{tabular}

Fonte: Brasil (1997).

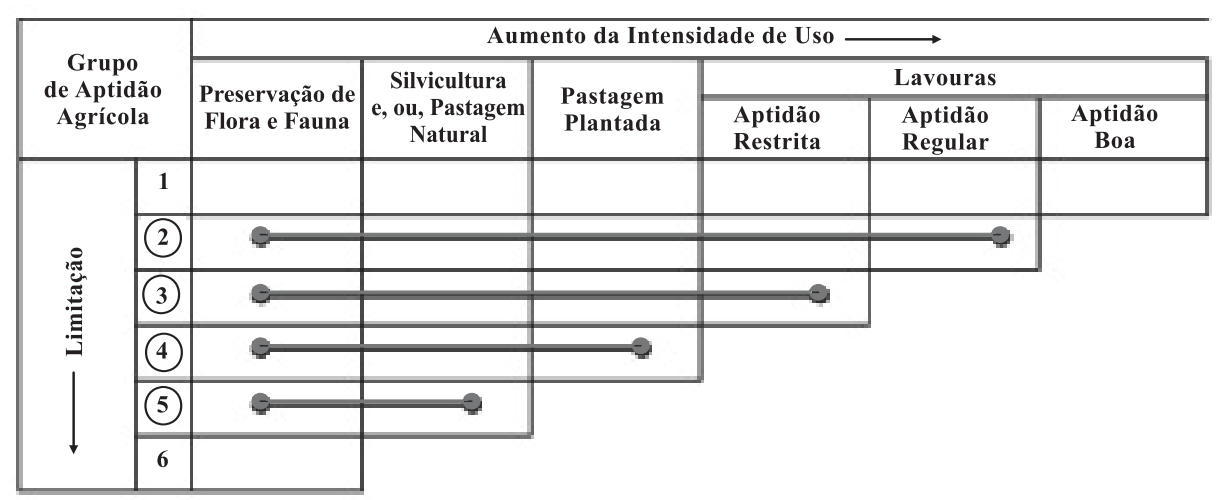

Figura 3. Alternativas de uso do solo conforme os graus de limitação, para cada grupo de aptidão agrícola. Fonte: adaptado de Ramalho \& Beek (1995). 
A simbologia das classes de aptidão agrícola apresenta letras que acompanham os algarismos e são indicativas das classes de aptidão (boa, regular ou restrita), conforme os níveis de manejo. O três níveis de manejo (a, b e c) referem-se à necessidade de diferentes níveis tecnológicos: primitivo, pouco desenvolvido e desenvolvido, respectivamente. Assim, o uso do solo depende dos níveis de manejo considerados.

\section{RESULTADOS E DISCUSSÃO}

\section{Critério de elegibilidade}

Os valores resultantes dos fatores inerentes do modelo USLE, da tolerância à perda de solo e do Risco Natural de Erosão (RNE) encontram-se na figura 4.

Os mapas de valores resultantes de RNE e do fator LS apresentam notável semelhança no delineamento das classes de valores. A matriz de correlação linear entre as variáveis RNE e o fator LS gerou o coeficiente de correlação (r) igual a 0,94 (ferramenta Band Collection Statistics, ESRI, 2006). Portanto, a topografia constitui significativa influência na determinação das áreas com elevados valores de RNE (Risse et al., 1993; Weill \& Sparovek, 2008) e, consequentemente, no delineamento de áreas Altamente Erodíveis (AE), com RNE maior que 8.

A aplicação do critério de elegibilidade restringiu as áreas de implantação do programa de PSA.
Considerando que o programa "Produtor de Água" é passível de ser aplicado em toda área de estudo, o critério de elegibilidade proposto foi capaz de beneficiar somente cerca de $39 \%$ da mesma área. Assim, a identificação de áreas com maior propensão natural à erosão é capaz de conduzir ao estabelecimento de áreas prioritárias para conservação do solo e água na bacia hidrográfica, além de favorecer o planejamento estratégico de investimentos. Essa metodologia de elegibilidade permite, também, a determinação da escala de prioridades para aplicação do PSA.

O cruzamento dos mapas de uso do solo e de classificação de áreas AE indica o grau de redução das áreas ocupadas por atividades agrícolas passíveis de serem contempladas pela nova proposta de PSA (Figura 5).

\section{Propostas de conservação}

Após a definição da base espacial de aplicação do programa de PSA, a área de estudo, bem como as áreas $\mathrm{AE}$, foram avaliadas mediante as características locais de aptidão agrícola, as quais apresentaram delineamento semelhante ao das classes de solo (Figura 6). Verifica-se que a região leste da bacia estudada apresenta maior aptidão para atividades de agricultura, enquanto a parte sul constitui menor aptidão para essas atividades. A parte norte, por sua vez, é inapta para atividades de agricultura, pois possui maior limitação de uso, restringindo-se às atividades de pecuária e silvicultura. Verifica-se que o atual padrão de ocupação do solo encontra-se em conformidade com as indicações de aptidão agrícola.

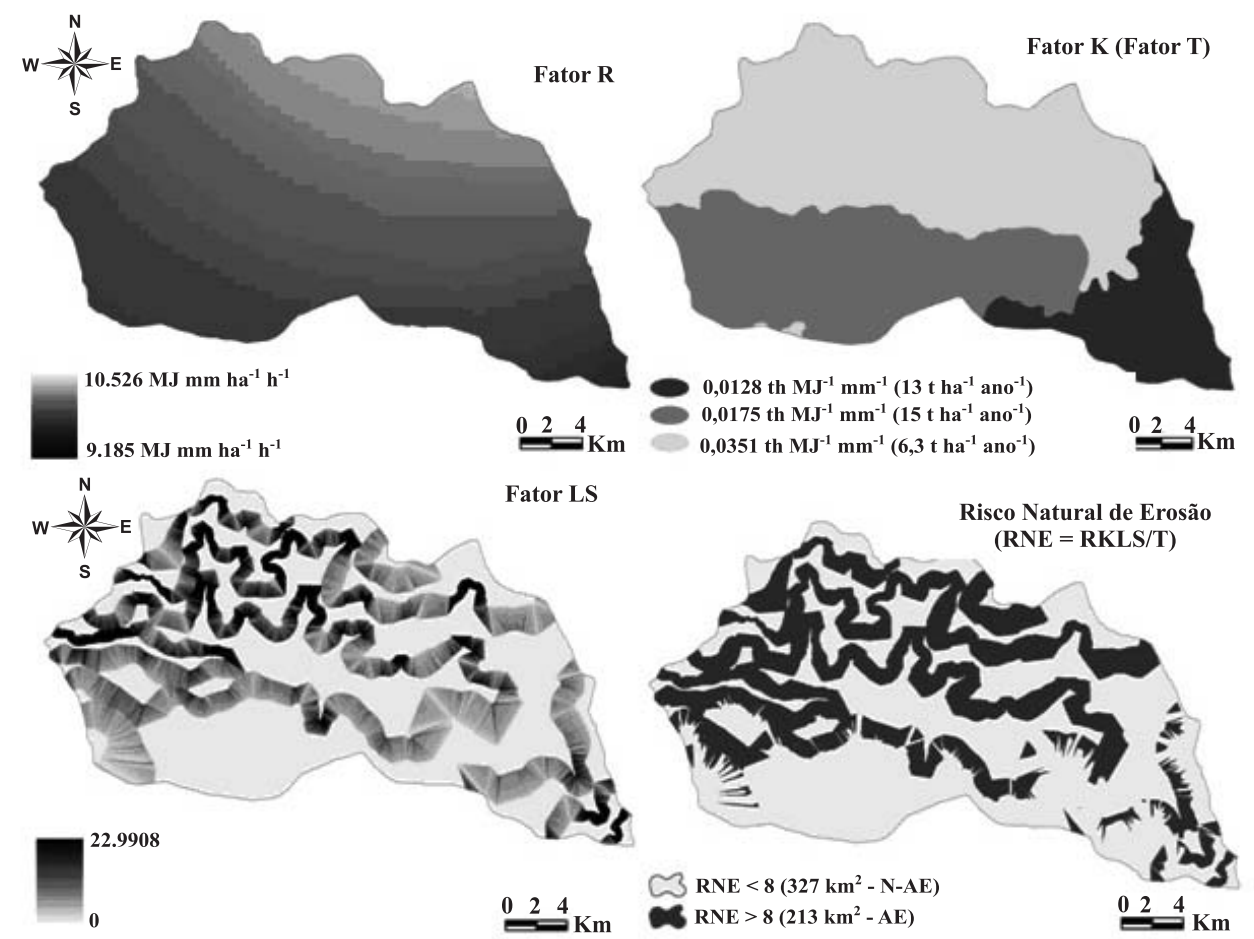

Figura 4. Valores resultantes dos fatores do modelo USLE e do índice RNE. 


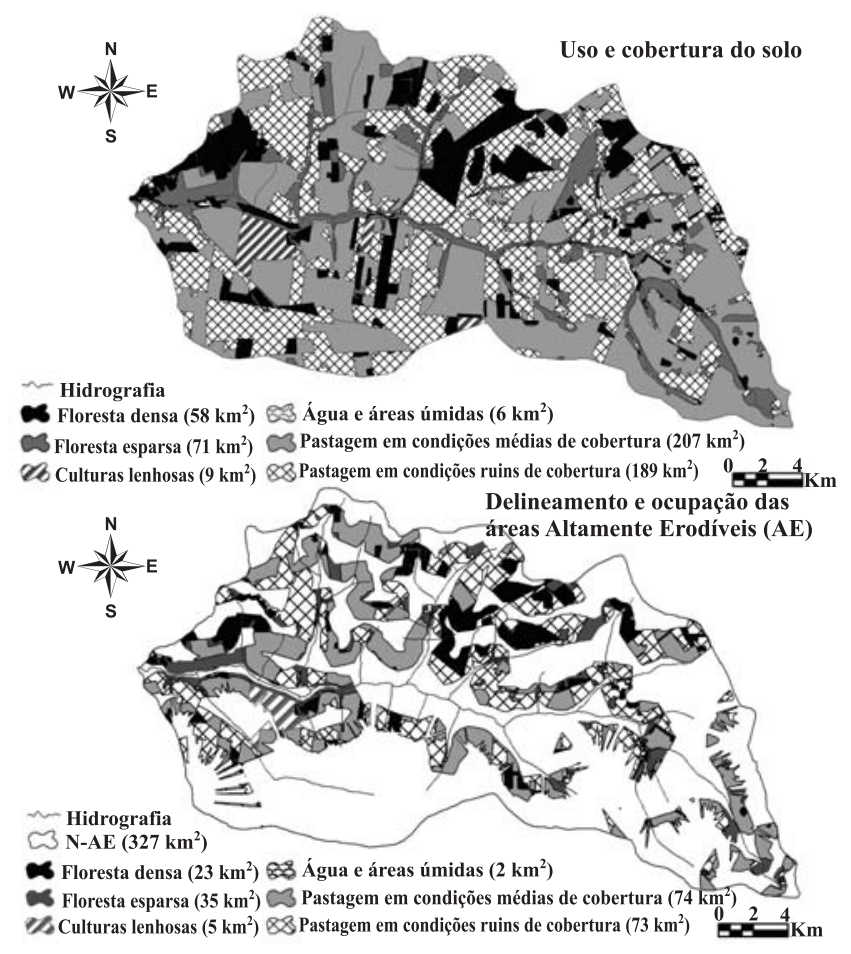

Figura 5. (A) uso e cobertura do solo; (B) delineamento e ocupação das áreas classificadas como Altamente Erodiveis (AE).
A nova abordagem do programa "Produtor de Água" possibilita, de forma satisfatória, essencialmente duas adaptações no programa original: direcionamento dos recursos financeiros a proprietários que possuem áreas com maior propensão natural à erosão; e análise ou elaboração de projetos de PSA conforme as características de aptidão agrícola da área. Nesse contexto, os fatores do modelo USLE (R, K, L e S), a tolerância à perda de solo e a classificação de aptidão agrícola atuam como importantes ferramentas, sendo relevante a estimativa in situ dessa caracterização, como o fator de erodibilidade e a tolerância a perda de solo (Li et al., 2009). Além disso, o incremento de pesquisas sobre erosão do solo na região de aplicação do PSA é importante no sentido de estabelecer detalhes das propostas de conservação a serem implementadas, tal como ocorre nos Estados Unidos (Renschler \& Harbor, 2002).

\section{CONCLUSÕES}

1. O critério de elegibilidade, baseado em elevados valores de risco natural à erosão, conduz ao estabelecimento de áreas prioritárias para conservação do solo e água e favorece o planejamento estratégico

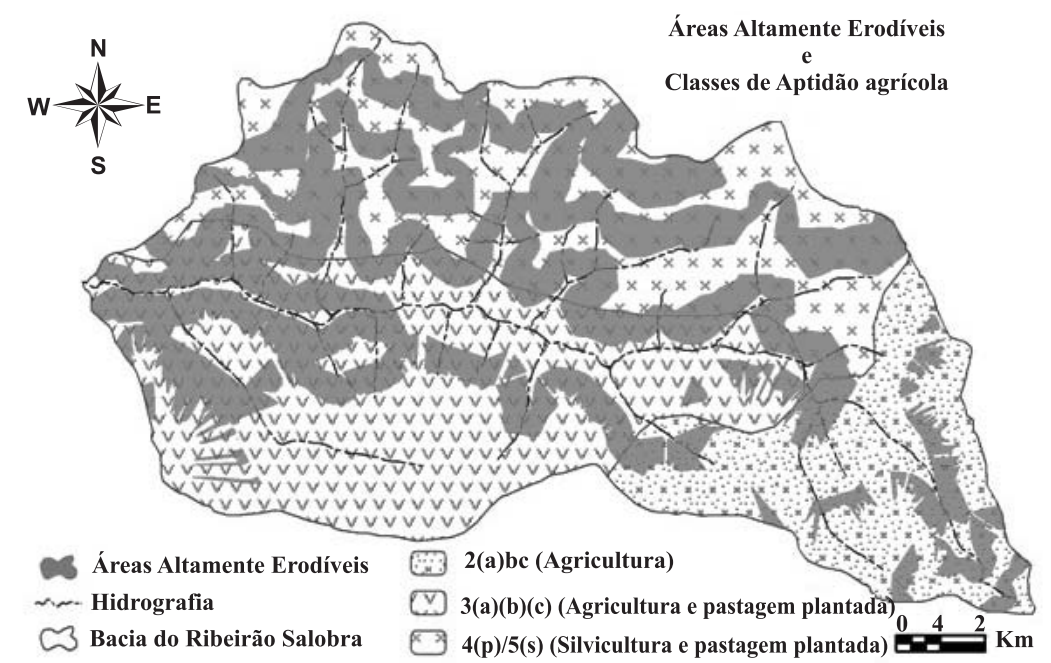

Figura 6. Distribuição das classes de aptidão agrícola e alternativas de uso intensivo do solo para projetos de implantação de PSA dentro das áreas classificadas como Altamente Erodíveis (AE).

Analisando a distribuição espacial das classes de aptidão agrícola e as áreas classificadas como $\mathrm{AE}$, identifica-se que $51,1 \%$ das áreas classificadas como AE estão localizadas em áreas inaptas para agricultura (porção norte da área de estudo), o que mostra a compatibilidade de informações, as quais apontam a suscetibilidade à erosão desses locais e a necessidade de cuidados especiais ou técnicas avançadas de conservação. Assim, torna-se evidente que a união desses aspectos reduz as alternativas de uso intensivo do solo, direcionando-as para a atividade pecuária. da bacia hidrográfica. Além disso, esse método de elegibilidade permite, também, a determinação de uma escala de prioridades para aplicação do PSA.

2. A aplicação da classificação de aptidão agrícola na parcela de áreas elegíveis reduziu drasticamente as opções de uso intensivo do solo. Em particular, a presente área de estudo, localizada próxima ao pantanal brasileiro, foi direcionada, em grande parte, para a atividade pecuária.

3. Apesar das limitações do modelo USLE, dos valores de tolerância à perda de solo e da subjetividade 
da classificação de aptidão agrícola, eles podem ser utilizados de forma qualitativa para norteamento do planejamento de conservação do solo e água, bem como na aplicação do PSA.

4. A análise das duas propostas de inovação do programa "Produtor de Água" permite inferir quanto à potencialidade delas na formação de estratégias de aplicação do programa, além de promover uso mais eficiente dos recursos financeiros destinados ao pagamento por serviços ambientais no contexto brasileiro.

\section{AGRADECIMENTOS}

Ao Conselho Nacional de Desenvolvimento Científico e Tecnológico (CNPq) e à Fundação de Apoio ao Desenvolvimento do Ensino, Ciência e Tecnologia do Estado de Mato Grosso do Sul (FUNDECT), pelo apoio financeiro; e aos revisores anônimos, pelas sugestões.

\section{LITERATURA CITADA}

AGÊNCIA NACIONAL DE ÁGUAS - ANA. Disponível em: <www.ana.gov.br/produagua>. Acesso em: jan. de 2011.

AGÊNCIA NACIONAL DE ÁGUAS - ANA. Manual operativo do Programa Produtor de Água. Superintendência de Usos Múltiplos. Brasília, ANA, 2008.

BASIC, F.; KISIC, I.; MESIC, M.; NESTROY, O. \& BUTORAC, A. Tillage and crop management effects on soil erosion in central Croatia. Soil Tillage Res., 78:197-206, 2004.

BOELLSTORFF, D. \& BENITO, G. Impacts of set-aside policy on the risk of soil erosion in central Spain. Agric. Ecosyst. Environ., 107:231-243, 2005.

BRASIL - Ministério das Minas e Energia. Projeto RADAMBRASIL. Folha SF.21 Campo Grande: Geologia, geomorfologia, pedologia, vegetação e uso potencial da terra. Rio de Janeiro, Levantamento de Recursos Naturais, 1982. 412p.

BRASIL. Projeto de Lei no 5.487. Institui a Política Nacional dos Serviços Ambientais, o Programa Federal de Pagamento por Serviços Ambientais e estabelece formas de controle e financiamento desse Programa. Brasília, 01 de julho de 2009. Disponível em: <http:// www.camara.gov.br/sileg/Prop_Detalhe.asp?id=439941>. Acesso em jan de 2011.

BRASIL. Ministério do Meio Ambiente, dos Recursos Hídricos e da Amazônia Legal. Programa Nacional de Meio Ambiente. Plano de Conservação da Bacia do Alto Paraguai- PCBAP. Brasília, PNMA, 1997.

BÜHLMANN, E.; WOLFGRAMM, B.; MASELLI, D.; HURNI, H.; SANGINOV, S.R. \& LINIGER, H.P. Geographic information system-based decision support for soil conservation planning in Tajikistan. J. Soil Water Conserv., 65:151-159, 2010.
CHAGAS, C.S.; CARVALHO JUNIOR, W.; PEREIRA, N.R. \& FERNANDES FILHO, E.I. Aplicação de um sistema automatizado (ALES - Automated Land Evaluation System) na avaliação das terras das microrregiões de Chapecó e Xanxerê, Oeste Catarinense, para o cultivo de grãos. R. Bras. Ci. Solo, 30:509-522, 2006.

CHAVES, H.M.L.; BRAGA JR., B.; DOMINGUES, A.F. \& SANTOS, D.G. Quantificação dos benefícios ambientais e compensações financeiras do "Programa do Produtor de Água" (ANA): I.Teoria. R. Bras. Rec. Hídricos, 9:5-14, 2004.

CHEN, X.; LUPI, F.; HE, G. \& LIU, J. Linking social norms to efficient conservation investment in payments for ecosystem services. PNAS, 106:11812-11817, 2009.

COSTA, G.P.D.; SOUZA, J.L.M.D. \& JESUS, M.R.G.D. Contraste entre duas metodologias de determinação do potencial agrícola das terras nas vilas rurais no município de Rio Negro, Estado do Paraná. Acta Sci. Agron., 30:687$695,2008$.

COSTANZA, R.; D'ARGE, R.; DE GROOT, R.; FARBER, S.; GRASSO, M.; HANNON, B.; LIMBURG, K.; NAEEM, S.; O'NEILL, R.V.; PARUEDO, J.; RASKIN, R.G.; SUTTON, P. \& van den BELT, M. The value of the world's ecosystem services and natural capital. Nature, 15:253-260, 1997.

COSTANZA, R.; D'ARGE, R.; DE GROOT, R.; FARBER, S.; GRASSO, M.; HANNON, B.; LIMBURG, K.; NAEEM, S.; O'NEILL, R.V.; PARUEDO, J.; RASKIN, R.G.; SUTTON, P. \& van den BELT, M. The value of the world's ecosystem services and natural capital. Ecol. Econ., 25:3-15, 1998.

DESMET, P.J.J. \& GOVERS, G. A GIS-procedure for automatically calculating the USLE LS-factor on topographically complex landscape units. J. Soil Water Conserv., 51:427-433, 1996.

ENGEL, S.; PAGIOLA, S. \& WUNDER, S. Designing payments for environmental services in theory and practice: An overview of the issues. Ecol. Econ., 65:663-674, 2008.

ENVIRONMENTAL SYSTEMS RESEARCH INSTITUTE ESRI. ArcGIS Professional GIS for the desktop, version 9.2. Redlands, 2006.

FOURNIER, F. The effect of climatic factors on soil erosion estimates of solids transported in suspension in runoff. Association Hydrologic Int. Public, 1956. 6p.

FSA - Food Security Act of 1985. United States, Public Law 99 - 198, Title XII: Conservation. 99 Stat. 1504, 23 dec. 1985.

GOMES, N.M.; FARIA, M.A.; SILVA, A.M.; MELLO, C.R. \& VIOLA, M.R. Variabilidade espacial de atributos físicos do solo associada ao uso e ocupação da paisagem. R. Bras. Eng. Agríc. Amb., 11:427-435, 2007.

INSTITUTO NACIONAL DE PESQUISAS ESPACIAIS INPE. Imagem LANDSAT 5 Thematic Mapper (TM), canais $1,2,3,4,5,6,7$, órbita 225 , ponto 74 , de 18 dezembro 2008. São José dos Campos Disponível em: http:// www.dgi.inpe.br/CDSR/. Acesso em: 17 mar 2009.

KINNELL, P.I.A. Event soil loss, runoff and the universal soil loss equation family of models: A review. J. Hydrol., 385:384-397, 2010. 
KOSOY, N.; MARTINEZ-TUMA, M.; MURADIAN, R. \& MARTINEZ-ALIER, J. Payments for environmental services in watersheds: Insights from a comparative study of three cases in Central America. Ecol. Econ., 61:446$455,2007$.

LANT, C.L.; KRAFT, S.E.; BEAULIEU, J.; BENNETT, D.; LOFTUS, T. \& NICKLOW, J. Using GIS-based ecologicaleconomic modeling to evaluate policies affecting agricultural watersheds. Ecol. Econ., 55:467-484, 2005.

LI, L.; DU, S.; WU, L. \& LIU, G. An overview of soil loss tolerance. Catena, 78:93-99, 2009

LOMBARDI NETO, F. Rainfall erosivity - its distribution and relationship with soil loss at Campinas, Brazil. West Lafayette, Purdue University, 1977. 53p. (Tese de Mestrado)

MENEZES, M.D.; CURI, N.; MARQUES, J.J.; MELLO, C.R. \& ARAÚJO, A.R. Levantamento pedológico e sistema de informações geográficas na avaliação do uso das terras em sub-bacia hidrográfica de minas gerais. Ci. Agrotec., 33:1544-1553, 2009 .

OLIVEIRA, A.; PINTO, S. \& LOMBARDi NETO, F. Caracterização de indicadores da erosão do solo em bacias hidrográficas com o suporte de geotecnologias e modelo preditivo. Est. Geogr., 5:63-86, 2007.

OLIVEIRA, F.P.; SANTOS, D.; SILVA, I.F. \& SILVA, M.L.N Tolerância de perda de solo por erosão para o Estado da Paraíba. R. Biol. Ci. Terra, 8:60-71, 2008.

OLIVEIRA, P.T.S.; ALVES SOBRINHO, T.; RODRIGUES, D.B.B. \& PANACHUKI, E. Erosion risk mapping applied to environmental zoning. Water Res. Manag., 25:10211036, 2011.

OLIVEIRA, P.T.S.; RODRIGUES, D.B.B.; ALVES SOBRINHO, T. \& PANACHUKI, E. Estimativa do fator topográfico da USLE a partir de três algoritimos. Ambi-Agua, 5:217-225, 2010 .

PEDRON, F.D.A.; POELKING, E.L.; DALMOLIN, R.S.D.; AZEVEDO, A.C.D.; \& KLANT, E. A aptidão de uso da terra como base para o planejamento da utilização dos recursos naturais no município de São João do Polêsine RS. Ci. Rural, 36:105-112, 2006.

POSTEL, S.L. \& THOMPSON, B.H. Watershed protection: Capturing the benefits of nature's water supply services. Nat. Res. Forum, 29:98-108, 2005.
RAMALHO FILHO, A. \& BEEK, K.J. Sistema de avaliação da aptidão agrícola das terras. 3.ed. Rio de Janeiro, EmbrapaCNPS, 1995. 65p.

RENSCHLER, C.S. \& HARBOR, J. Soil erosion assessment tools from point to regional scales-the role of geomorphologists in land management research and implementation. Geomorphology, 47:189-209, 2002.

RISSE, L.M.; NEARING, M.A.; NICKS, A.D. \& LÂ̂EN, J.M. Error assessment in the universal soil loss equation. Soil Sci. Soc. Am. J., 57:825-833, 1993.

SCHIETTECATTE, W.; D’HONDT, L.; CORNELIS, W.M.; ACOSTA, M.L.; LEAL, Z.; LAUWERS, N.; ALMOZA, Y.; ALONSO, G.R.; DÍAS, J.; RUÍZ, M. \& GABRIELS, D. Influence of landuse on soil erosion riskin the Cuyaguateje watershed (Cuba). Catena, 74:1-12, 2008.

SEIDL, A.F.; SILVA, J.S.V. \& MORAES, A.S. Cattle ranching and deforestation in the Brazilian Pantanal. Ecol. Econ., $36: 413-425,2001$

SPAROVEK, G. \& DE MARIA, I.C. Multiperspective analysis of erosion tolerance. Sci Agric, 60:409-416, 2003.

UNITED STATES DEPARTAMENT OF AGRICULTURE NATIONAL RESOURCES CONSERVATION SERVICE - USDA-NRCS. National Food Security Act Manual (NFSAM): Highly Erodible Determinations. 4.ed Washington, U.S. Dept. of Agriculture, Soil Conservation Service,. 2006. Parte 511. p.1-22.

WANTZEN, K.M.; SANTOS, S.A.; DA CUNHA, C.N.; JUNK, W.J.; GIRARD, P.; ROSSETTO, O.C.; PENHA, J.M.; COUTO, E.G.; BECKER, M.; PRIANTE, G.; TOMAS, W.M.; SANTOS, S.A.; MARTA, J.; DOMINGOS, I.; SONODA, F.; CURVO, M. \& CALLIL, C. Towards a sustainable management concept for ecosystem services of the Pantanal wetland. Ecohydrol. Hydrobiol., 8:115-138, 2008.

WEILL, M.A.M. \& SPAROVEK, G. Estudo da erosão na microbacia do Ceveiro (Piracicaba, SP): I - Estimativa das taxas de perda de solo e estudo de sensibilidade dos fatores do modelo EUPS. R. Bras. Ci Solo, 32:801-814, 2008.

WISCHMEIER, W.H. \& SMITH, D.D. Predicting rainfall erosion losses. A guide to conservation planning. Washington, USDA, 1978. 58p. (Agriculture Handbook, 537) 